\title{
Preservation HIV-1-Specific IFN $\gamma+$ CD4+ T-Cell Responses in Breakthrough Infections After Exposure to Tenofovir Gel in the CAPRISA 004 Microbicide Trial
}

\author{
Marianne W. Mureithi, PhD, *† Danielle Poole, MPH, *† Vivek Naranbhai, MD, * $\neq$ \\ Shabashini Reddy, MSc, * Nompumelelo P. Mkhwanazi, MSc, * Sengeziwe Sibeko, MBChB, MSc, t \\ Lise Werner, MSc, $\neq$ Quarraisha Abdool Karim, PhD, $\$$ Salim Abdool Karim, MBChB, PhD, $\$$ \\ Thumbi Ndung'u, PhD, *广 Marcus Altfeld, MD, PhD, *† and The CAPRISA004 Trial Group
}

\begin{abstract}
The Centre for the AIDS Program of Research in South Africa 004 trial demonstrated reduction of sexual HIV-1 acquisition in women using a vaginal microbicide containing tenofovir. A better understanding of the consequences of antiretroviral-containing microbicides for immune responses in individuals with intercurrent HIV-1 infection is needed for future trials combining the use of microbicides with HIV-1 vaccines. Investigation of immune responses in women who acquired HIV-1 although using tenofovir
\end{abstract}

Received for publication October 24, 2011; accepted January 31, 2012.

From the *HIV Pathogenesis Program, Doris Duke Medical Research Institute and KwaZulu-Natal Research Institute for TB and HIV (KRITH), Nelson R. Mandela School of Medicine, University of KwaZulu-Natal, Durban, South Africa; †Ragon Institute of Massachusetts General Hospital, MIT, and Harvard, Charlestown, MA; + Centre for the AIDS Program of Research in South Africa (CAPRISA), University of KwaZulu-Natal, Durban, South Africa; and §Department of Epidemiology, Columbia University, New York, NY.

Supported through the KwaZulu-Natal Research Institute for TB and HIV (K-RITH) and by a grant from the Doris Duke Charitable Foundation to MA. The parent trial (CAPRISA 004) was supported by the United States Agency for International Development (USAID), FHI360 [USAID co-operative agreement \# GPO-A-00-05-00022-00, contract \# 132119], and the Technology Innovation Agency (LIFElab) of the South African government's Department of Science \& Technology Tenofovir was provided by Gilead Sciences and the gel was manufactured and supplied for the CAPRISA 004 trial by CONRAD. The current studies are part of the CAPRISA TRAPS (Tenofovir gel Research for AIDS Prevention Science) Program, which is funded by CONRAD, Eastern Virginia Medical School [USAID co-operative grant \#GP00-08-00005-00, subproject agreement \# PPA-09-046]. The views expressed by the authors do not necessarily reflect the views of USAID, Gilead Sciences, Eastern Virginia Medical School or CONRAD.

The results from this study were presented in an oral presentation at the AIDS Vaccine meeting, 2011, Bangkok, Thailand.

The authors Q.A.K. and S.S.A.K. are coinventors with investigators from Gilead Sciences on two pending patents on tenofovir gel. The authors S.S.A.K. once received $\$ 2500$ for serving on an Advisory Panel on PrEP for Merck. M.A. once received $\$ 1500$ as a CME Honorarium from Gilead Science. M.W.M. was supported by the National Institutes of Health Office of the Director, Fogarty International Center, through the International Clinical Research Fellows Program at Vanderbilt (R24 TW007988).

The authors have no other conflicts of interest to disclose.

Correspondence to: Marcus Altfeld, MD, Associate Professor, Harvard Medical School, Ragon Institute of Massachusetts General Hospital, MIT, and Harvard, 149 13th Street, Charlestown, MA 02129 (e-mail: maltfeld@partners.org).

Copyright (C 2012 by Lippincott Williams \& Wilkins gel showed significantly higher $(P=0.01)$ Gag-specific IFN $\gamma+$ CD4+ T-cell responses. The use of tenofovir-containing gel around the time of infection can modulate HIV-1 immunity, and these immunological changes need to be considered in future trials combining vaccines and microbicides.

Key Words: HIV-1, vaginal microbicide, tenofovir, HIV-1-specific CD4+ T-cell help

(J Acquir Immune Defic Syndr 2012;60:124-127)

\section{INTRODUCTION}

A recent randomized controlled trial undertaken by the Centre for the AIDS Program of Research in South Africa (CAPRISA) reported that a vaginal microbicide gel containing $1 \%$ tenofovir reduced the risk of HIV-1 infection in women by $39 \% .^{1}$ Although confirmation of these findings in additional studies are needed and similar studies are currently underway, these results will have significant implication for the design of future prevention trials, including vaccine trials in which both vaccinees and placebo recipients might receive prophylactic antiretroviral drugs in gel or oral formulation. ${ }^{2}$ A better understanding of the consequences of antiretroviral-containing microbicides for immune responses in individuals with intercurrent HIV-1 infection is therefore critical to take these immunological changes into consideration in the design of future trials combining the use of microbicides with HIV-1 vaccines. ${ }^{2}$ In the present study, we investigated innate and adaptive immune responses during primary HIV-1 infection in women who acquired HIV-1 although using either tenofovir gel or placebo in the CAPRISA004 trial.

\section{MATERIALS AND METHODS}

\section{Study Population}

Cryopreserved peripheral blood mononuclear cell (PBMCs) were collected from sexually active HIV-1 clade C-infected 18-year-old to 40-year-old women in urban and rural KwaZulu-Natal, South Africa, enrolled in CAPRISA $004 .{ }^{1}$ The eligibility and exclusion criteria for the parent trial have been previously reported. ${ }^{1}$ Participants who 
acquired HIV stopped using study gel on confirmation of HIV infection, as per the trial protocol. ${ }^{1}$ From the total of 98 intercurrent HIV infections that occurred, 36 randomly selected HIV-1-infected female adults exposed either to tenofovir gel $(n=17)$ or placebo $(n=19)$ were selected for this substudy. The study was approved by the University of KwaZulu-Natal and Massachusetts General Hospital Biomedical Research Ethics Committee, Family Health International Protection of Human Subjects Committee, and the South African Medicines Control Council with each subject giving informed consent for participation.

\section{Characterization of Phenotype and Function of Innate and Adaptive Immune Cells Using Multiparameter Flow Cytometry}

The phenotypic characteristics of natural killer (NK) cells and myeloid dendritic cells (mDCs) were assessed by multiparameter flow cytometry using cyropreserved PBMC collected within 3 months of HIV-1 infection. After gating on lineage negative (CD3neg, CD19neg, CD56neg) lymphocytes, anti-CD11c was used to identify mDCs, as described. ${ }^{3,4}$ Antibodies directed against Human Leukocyte Antigen (HLA)-DR, CD83, and CD86 were used to study the activation and maturation stage of mDCs directly ex-vivo. NK cells were defined as CD3negCD56/CD16+ cells, and antibodies directed against HLA-DR, CD38, and CD69 were used to study the activation status of NK cells, as described. ${ }^{5}$

Intracellular measurement of interferon gamma (IFN- $\gamma$ ), tumor necrosis factor alpha (TNF- $\alpha$ ), and interleukin 2 (IL-2) production by $\mathrm{CD} 4+$ and $\mathrm{CD} 8+\mathrm{T}$ cells was undertaken as described. ${ }^{6,7}$ Cryopreserved PBMCs were stimulated for 6 hours at $37^{\circ} \mathrm{C} ; 5 \% \mathrm{CO}_{2}$ with Gag and Nef peptide pools spanning the HIV-1 clade $\mathrm{C}$ consensus sequence (final concentration $2 \mu \mathrm{g} / \mathrm{ml}$ per peptide) in the presence of brefeldin A. Negative controls with PBMCs and media alone and positive controls stimulated with phytohemaggultinin were included in the assays. Between 500,000-1,000,000 events were acquired on a LSRII flow cytometer, and data analysis was performed using FlowJo version 8.8.2 (TreeStar, Inc). Boolean gating was used to create a full array of the 8 possible response patterns when testing 3 functions, and data were further analyzed using SPICE 5 and PESTLE software programs (kindly provided by Drs Roederer and Nozzi, National Institute of Allergy and Infectious Diseases, National Institutes of Health).

\section{Statistical Analyses}

Statistical analyses and graphical presentation was done using Graphpad Prism 5 (Graphpad). Results are given as averages with standard deviations. Paired 2-tailed Student $t$ tests were used to test statistical significance. Differences after comparisons were considered statistically significant if $P<0.05$.

\section{RESULTS}

\section{Characteristics of Study Subjects}

Cryopreserved PBMCs collected from 36 sexually active HIV-1-infected women in urban and rural KwaZulu-

\begin{tabular}{|c|c|c|c|}
\hline & Tenofovir & Placebo & $P$ \\
\hline $\mathrm{n}$ & 17 & 19 & - \\
\hline Age & $24(19-37)$ & $23(19-31)$ & 0.69 \\
\hline Days post infection & $85(20-345)$ & $64(15-481)$ & 0.30 \\
\hline Initial CD4+ T-cell count & $454(182-955)$ & $556(240-1036)$ & 0.17 \\
\hline $\begin{array}{l}\text { CD4+ T-cell count } \\
\text { post infection }\end{array}$ & 464 (197-955) & $510(240-1036)$ & 0.11 \\
\hline Initial viral $\log (\log )$ & $4.66(2.60-6.53)$ & $4.42(2.60-5.85)$ & 0.72 \\
\hline $\begin{array}{l}\text { Viral load post } \\
\text { infection (log) }\end{array}$ & $4.96(2.60-5.86)$ & $4.51(2.60-5.51)$ & 0.58 \\
\hline
\end{tabular}

Average and range is shown.

Natal enrolled into the CAPRISA 004 trial $^{1}$ were randomly selected and studied. Investigators were blinded regarding the study group for the immunological analysis. Seventeen of these women were part of the tenofovir gel arm and 19 of the placebo arm. The demographic, immunological, and virological characteristics of the study cohort are presented in Table 1 . Women in the 2 arms did not differ in the days since infection, and HIV-1 viral loads and CD4+ T-cell counts at presentation or analysis, and did also not differ in the distribution of low, intermediate, and high adherers.

\section{Lack of Differences in Innate Immune Cells Between the Study Arms at 3 Months Post Infection}

We initially examined the frequencies and activation status of NK cells and mDCs between women in the tenofovir or placebo arms. The average frequencies of NK cells did not differ between the 2 arms (average NK cells $4.7 \% \pm 3.8 \%$ vs. $5.5 \% \pm 4.6 \%$ ). Furthermore, the average percentage of NK cells expressing HLA-DR $(12 \% \pm 6.3 \%$ vs. $12.9 \% \pm$ $9.2 \%), \operatorname{CD} 38(68.9 \% \pm 25.3 \%$ vs. $67.1 \% \pm 28.6 \%)$ or CD69 $(10.3 \% \pm 10.9 \%$ vs. $10.7 \% \pm 7.1 \%)$ did not differ between the women who received tenofovir compared with those who received placebo ( $P>0.05$ for all comparisons). Similarly, the average percentage of $\mathrm{mDCs}(5.7 \% \pm 3.2 \%$ vs. $5.9 \% \pm 1.9 \%$ ) and their activation status (HLA-DR: $19.8 \% \pm$ $19.5 \%$ vs. $17.5 \% \pm 11.3 \%$; CD $83: 6.8 \% \pm 9.5 \%$ vs. $6.7 \% \pm$ $4.2 \%$, and CD86: $19.3 \% \pm 21.4 \%$ vs. $11.5 \% \pm 11.7 \%$; $p>$ 0.05 for all comparisons) did not differ between tenofovir and placebo gel recipients. Taken together, these data demonstrate that frequencies and activation status of cells of the innate immune system were not significantly impacted by the use of tenofovir gel in women with breakthrough HIV-1 infection approximately 3 months after infection.

\section{Preservation of HIV-1-Specific IFN $\gamma$ CD4+ T Cells During Primary Infection}

Previous studies have shown that HIV-1 Gag and Nef are targeted by HIV-1-specific T cells during primary infection, and that virus-specific CD4+ T-cell responses are rapidly lost after infections. ${ }^{8}$ We compared HIV-1 clade C Gagspecific and Nef-specific CD4+ and CD8+ T-cell activity 
FIGURE 1. Higher HIV-1 Gag-specific and Nef-specific CD4+ T-cell responses in tenofovir recipients. The dot plot represents the median \% IFN $\gamma+$ CD4 ${ }^{+}$T cells in response to HIV-1 Gag (left panel) and Nef (right panel) in women enrolled in the tenofovir arm $(n=17)$ compared with women who received placebo $(n=19)$.
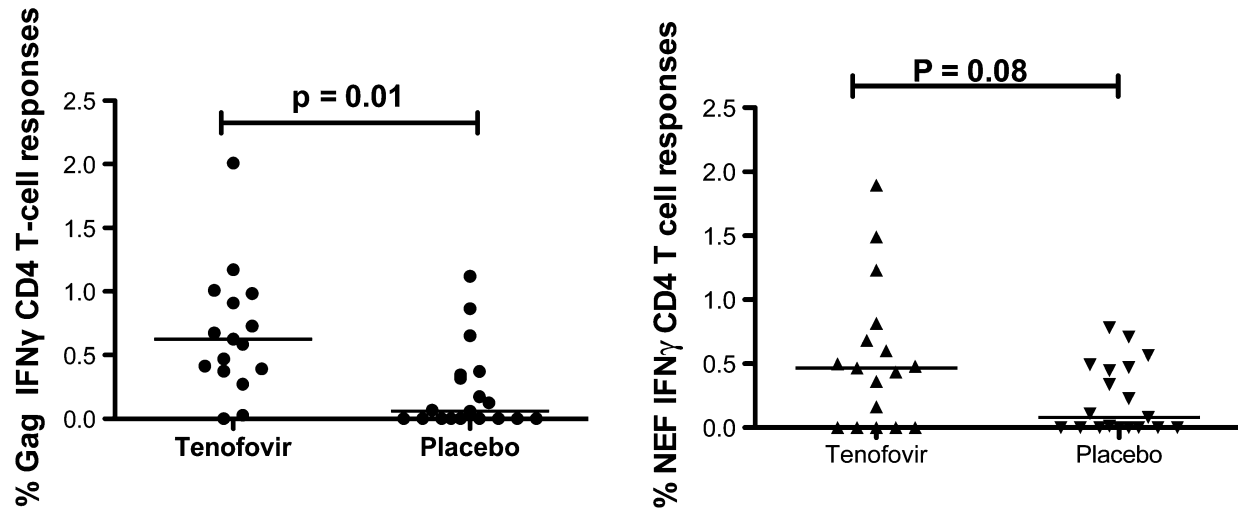

between women who received tenofovir gel or placebo recipients. As shown in Figure 1, Gag-specific IFN- $\gamma+$ CD4+ Tcell responses were significantly higher in the women in the tenofovir gel arm compared with placebo $(P=0.01)$. No correlation was observed between days postinfection and Gagspecific IFN- $\gamma+\mathrm{CD} 4+$ T-cell responses $(R=-0.2 ; P=0.3)$. HIV-1 Nef-specific CD4+ T-cell responses also trended to be higher in women in the tenofovir gel arm compared with placebo recipients $(P=0.08$, Fig. 1). No significant differences were observed in the percentage of Gag-specific and Nef-specific CD4+ T-cells producing TNF- $\alpha$ or IL-2 between the 2 groups (data not shown).

Gag-specific and Nef-specific IFN- $\gamma+$ CD8+ T-cell responses were also higher in the tenofovir arm compared with placebo though those differences did not reach statistical significance (Gag: average $1.1 \% \mathrm{IFN}-\gamma+\mathrm{CD} 8+\mathrm{T}$ cells $\pm 1.0 \%$ in tenofovir arm vs. $0.7 \% \pm 0.7 \%$ in placebo, $P=0.33$; Nef: average $1.1 \%$ IFN- $\gamma+\mathrm{CD} 4+\mathrm{T}$ cells \pm $1.4 \%$ in tenofovir arm vs. $0.35 \% \pm 0.5 \%$ in placebo, $P$ $=0.35$ ). Furthermore, Gag and Nef-specific CD8+ T cells IL-2 and TNF- $\alpha$ production did not differ significantly between the 2 groups (data not shown). We also assessed the polyfunctionality of the CD4+ and CD8+ T cells by the simultaneous quantification of three functions (IL-2, IFN- $\gamma$, and TNF- $\alpha$ production) in response to Gag and Nef stimulation and observed no significant differences in the polyfunctionality of the $\mathrm{CD} 4+$ and $\mathrm{CD} 8+\mathrm{T}$ cells in the tenofovir arm compared with placebo $(P>0.05)$. Overall, a vast majority of HIV-1-specific T cells were monofunctional $(>90 \%)$ in both the tenofovir gel and placebo group. Taken together, these data demonstrate that the HIV-1-specific CD8+ T-cell responses in women with breakthrough infection during tenofovir microbicide use did not significantly differ from those that used placebo, but that Gag-specific IFN $\gamma+\mathrm{CD} 4+\mathrm{T}$ cells responses were significantly higher in HIV-1-infected women randomized to the tenofovir gel arm.

\section{DISCUSSION}

The use of a tenofovir-containing vaginal microbicide gel for the prevention of HIV-1 infection in sexually active, HIV-1-uninfected adult women in KwaZulu-Natal, South Africa, showed a significant reduction of HIV-1 infection rates by
$39 \%$ in the primary intent-to-treat analysis. ${ }^{1}$ Here, we examined the innate and adaptive immune responses in women with breakthrough HIV-1 infection that either used tenofovir microbicide gel or were part of the placebo arm. The frequencies and activation status of principal effector cells of the innate immune response, including $\mathrm{NK}$ cells and mDCs, assessed within 3 months of HIV-1 infection were not affected by the use of the tenofovir gel. Previous studies have demonstrated a significant expansion of NK cells in infected individuals during the early phase of HIV-1 infection and a subsequent contraction of $\mathrm{NK}$ cell and $\mathrm{mDC}$ populations. $^{9-11}$ We can therefore not exclude that NK cell and $\mathrm{mDC}$ frequencies in the initial 3 months of infection might have been affected by the use of the tenofovir gel, and that these changes in innate effector cells might have subsequently contributed to the observed functional differences in HIV-1-specific CD4+ T cells. ${ }^{12}$

HIV-1-specific T cells are considered critical for the control of HIV-1 replication and disease progression. ${ }^{8,13-15}$ Although HIV-1-specific CD8+ T-cell responses to both Gag and Nef peptides did not significantly differ between the groups, HIV-1 Gag-specific IFN- $\gamma+\mathrm{CD} 4+\mathrm{T}$-cell responses were significantly higher in women in the tenofovir gel arm compared with the placebo arm. HIV$1-$ specific CD4 $+\mathrm{T}$ cell responses are rapidly lost after acute infection in the presence of continuing HIV-1 replication, and this is in contrast to HIV-1-specific CD8+ T-cell frequencies which tend to increase over at least the first year of HIV-1 infection with continues exposure to antigen. ${ }^{16-20}$ Previous studies have shown that CD4+ T cells and in particular gut-associated CD4+ T cells are severely depleted within the first few days/weeks of infection ${ }^{21}$ and that HIV-1 preferentially infects HIV-1-specific CD4+ T cells. ${ }^{22}$ The presence of an antiretroviral agent, such as tenofovir, during this crucial period might protect CD4 $+\mathrm{T}$ cells from deletion, allowing for a preservation of HIV1 -specific IFN- $\gamma \mathrm{CD} 4+\mathrm{T}$ cells. The observation that women receiving tenofovir gel maintained higher Gag-specific CD4+ T-cell responses despite the reported lack of differences in viral load set-point between tenofovir gel and placebo recipients ${ }^{1}$ was however unexpected, and the long-term clinical benefit of this preservation, and its consequences for HIV-1-specific immune function, will require further investigation. 
Taken together, our studies in a subset of women who experienced breakthrough HIV-1 infection despite randomization to tenofovir gel usage demonstrate no significant alteration in the frequencies and activation status of key innate immune cells and HIV-1-specific CD8+ T-cell responses 3 months after infection. However, tenofovir gel applied vaginally around the time of HIV-1 transmission might protect HIV-1-specific CD4+ T-cell responses in infected individuals. This study demonstrates for the first time that the use of vaginal microbicides containing antiretroviral drugs can modulate HIV-1-specific immunity in individuals with breakthrough infection. These consequences of microbicide use for immune responses in individuals that acquire HIV-1 have to be considered in the design of future trials that will combine microbicides with HIV-1 vaccines.

\section{REFERENCES}

1. Abdool Karim Q, Abdool Karim SS, Frohlich JA, et al. Effectiveness and safety of tenofovir gel, an antiretroviral microbicide, for the prevention of HIV infection in women. Science. 2010;329:1168-1174.

2. Excler JL, Rida W, Priddy FH, et al. AIDS vaccines and pre-exposure prophylaxis: is synergy possible? AIDS Res Hum Retroviruses. 2011;27: 669-680.

3. Meier A, Chang JJ, Chan ES, et al. Sex differences in the Toll-like receptor-mediated response of plasmacytoid dendritic cells to HIV-1. Nat Med. 2009;15:955-959.

4. Meier A, Fisher A, Sidhu HK, et al. Rapid loss of dendritic cell and monocyte responses to TLR ligands following venipuncture. J Immunol Methods. 2008;339:132-140.

5. Alter G, Suscovich TJ, Teigen N, et al. Single-stranded RNA derived from HIV-1 serves as a potent activator of NK cells. J Immunol. 2007; 178:7658-7666.

6. Mkhwanazi N, Thobakgale CF, van der Stok M, et al. Immunodominant HIV-1-specific HLA-B- and HLA-C-restricted CD8+ T cells do not differ in polyfunctionality. Virology. 405:483-491.

7. Thobakgale CF, Ramduth D, Reddy S, et al. Human immunodeficiency virus-specific CD8+ T-cell activity is detectable from birth in the majority of in utero-infected infants. J Virol. 2007;81:12775-12784.
8. Rosenberg ES, Altfeld M, Poon SH, et al. Immune control of HIV-1 after early treatment of acute infection. Nature. 2000;407:523-526.

9. Alter G, Teigen N, Ahern R, et al. Evolution of innate and adaptive effector cell functions during acute HIV-1 infection. J Infect Dis. 2007; 195:1452-1460.

10. Alter G, Teigen N, Davis BT, et al. Sequential deregulation of NK cell subset distribution and function starting in acute HIV-1 infection. Blood. 005;106:3366-3369.

11. Sabado RL, O'Brien M, Subedi A, et al. Evidence of dysregulation of dendritic cells in primary HIV infection. Blood. 2010;116:3839-3852.

12. Pacanowski J, Kahi S, Baillet M, et al. Reduced blood CD123+ (lymphoid) and CD11c+ (myeloid) dendritic cell numbers in primary HIV-1 infection. Blood. 2001;98:3016-3021.

13. Deeks SG, Walker BD. The immune response to AIDS virus infection: good, bad, or both? J Clin Invest. 2004;113:808-810.

14. Virgin HW, Walker BD. Immunology and the elusive AIDS vaccine. Nature. 2010;464:224-231.

15. Gloster SE, Newton P, Cornforth D, et al. Association of strong virusspecific CD4 $\mathrm{T}$ cell responses with efficient natural control of primary HIV-1 infection. AIDS. 2004;18:749-755.

16. Altfeld M, Rosenberg ES, Shankarappa R, et al. Cellular immune responses and viral diversity in individuals treated during acute and early HIV-1 infection. $J$ Exp Med. 2001;193:169-180.

17. Lichterfeld M, Kaufmann DE, Yu XG, et al. Loss of HIV-1-specific CD8+ $\mathrm{T}$ cell proliferation after acute HIV-1 infection and restoration by vaccine-induced HIV-1-specific CD4+ T cells. J Exp Med. 2004;200: $701-712$.

18. Oxenius A, Price DA, Trkola A, et al. Loss of viral control in early HIV-1 infection is temporally associated with sequential escape from CD8+ T cell responses and decrease in HIV-1-specific CD4+ and CD8+ T cell frequencies. J Infect Dis. 2004;190:713-721.

19. Streeck H, Jolin JS, Qi Y, et al. Human immunodeficiency virus type 1 -specific CD8+ T-cell responses during primary infection are major determinants of the viral set point and loss of CD4+ T cells. $J$ Virol. 2009;83:7641-7648.

20. Streeck H, Nixon DF. T cell immunity in acute HIV-1 infection. J Infect Dis. 2010;202(suppl 2):S302-S308.

21. Brenchley JM, Schacker TW, Ruff LE, et al. CD4+ T cell depletion during all stages of HIV disease occurs predominantly in the gastrointestinal tract. J Exp Med. 2004;200:749-759.

22. Douek DC, Brenchley JM, Betts MR, et al. HIV preferentially infects HIV-specific CD4+ T cells. Nature. 2002;417:95-98. 